\title{
Hierarchical Modeling of Heterogeneous Plates ${ }^{1}$
}

A.C. CARIUS, 2 A.L. MADUREIRA, ${ }^{3}$ Laboratório Nacional de Computação Científica, Av. Getúlio Vargas, 333, 25651-070 Petrópolis, RJ, Brazil.

\begin{abstract}
We describe here the modeling of highly heterogeneous plates, when three different lenght scales are present: the area of the middle surface, the plate thickness, and the heterogeneity scale. We derive a two-dimensional PDE model for such problem, which turns out to have rough coefficients. We employ asymptotic techniques to estimate the modeling error with respect to the thickness. To tame the numerical troubles of the resulting model we use finite elements methods of multiscale type.
\end{abstract}

\section{Introduction}

The first step to model plate problems is to perform some sort of dimension reduction, and approximate a three-dimensional problem with a two-dimensional one. Most likely the most common arguments to obtain plate models are based on physical properties of the underlying problem, often combined with some mathematical reasoning. It is also possible to derive the models using asymptotic techniques, usually with a sound mathematical basis. The asymptotic arguments consist in taking the plate thickness to zero and finding "limit problems." For instance, linearly elastic plates have as limit biharmonic equations [8]. For heterogeneous materials however, an extra issue arises. There are situations when the attempts of homogenizing the material may lead to different models, depending on which limit is considered first, i.e., homogenization first and then dimension reduction, or the other way around $[9]$.

To avoid such undesirable peculiarity, we shall use hierarchical modeling. In such approach, the solution can defined as critical points of certain functionals, in the subspace of functions that are polynomials in the transverse direction.

In this work we consider the Poisson equation in a heterogeneous plate of thickness $2 \delta$ given by $P^{\delta}=\Omega \times(-\delta, \delta)$, where $\Omega \subset \mathbb{R}^{2}$ is a bounded open domain with Lipschitz boundary $\partial \Omega$. Let $\partial P_{L}^{\delta}=\partial \Omega \times(-\delta, \delta)$ be the lateral side of the plate, and $\partial P_{ \pm}^{\delta}=\Omega \times\{-\delta, \delta\}$ its top and bottom. We denote a typical point of $P^{\delta}$ by $\underline{x}=\left(\underset{\sim}{x}, x_{3}\right)$, where $\underset{\sim}{x}=\left(x_{1}, x_{2}\right) \in \Omega$. Accordingly, we write

\footnotetext{
${ }^{1}$ The first author had support from CAPES, and the second author was partially supported by the CNPq/Brazil Projects 306104/2004-0 and 486026/2006-0, and also by FAPERJ Project APQ1 E-26/170.629/2006.

${ }^{2}$ carol@lncc.br

3 alm@lncc.br
} 
$\nabla=\left(\underset{\sim}{\nabla}, \partial_{3}\right)=\left(\partial_{1}, \partial_{2}, \partial_{3}\right)$, where $\partial_{i}$ indicates the partial derivative with respect to $\bar{x}_{i}$. Also, $\partial_{i j}=\partial_{i} \partial_{j}$.

Let $u^{\delta} \in H^{1}\left(P^{\delta}\right)$ be the weak solution of

$$
\begin{gathered}
-\operatorname{div}\left(\stackrel{A}{\underline{A}} \underline{\nabla} u^{\delta}\right)=f^{\delta} \quad \text { in } P^{\delta} \\
u^{\delta}=0 \quad \text { in } \partial P_{L}^{\delta}, \quad \frac{\partial u^{\delta}}{\partial n}=g^{\delta} \quad \text { in } \partial P_{ \pm}^{\delta},
\end{gathered}
$$

where $f^{\delta}: P^{\delta} \rightarrow \mathbb{R}$ and $g^{\delta}: \partial P_{ \pm}^{\delta} \rightarrow \mathbb{R}$. The matrix $\underset{=}{A}: P^{\delta} \rightarrow \mathbb{R}_{\mathrm{SYM}}^{3 \times 3}$ is such that

$$
\underline{A}(\underline{x})=\left(\begin{array}{cc}
\underset{\sim}{a} \underset{\sim}{(x)} & 0 \\
0 & a_{33}(\underset{\sim}{x})
\end{array}\right),
$$

where $\underset{\approx}{a}: \Omega \rightarrow \mathbb{R}_{\mathrm{SYM}}^{2 \times 2}$, and $a_{33}: \Omega \rightarrow \mathbb{R}$. We also assume that $a_{i j}, f^{\delta}$, and $g^{\delta}$ are $C^{\infty}$ functions, and that there exist constants $\alpha$ and $\beta$ such that

$$
\alpha\|\underline{\xi}\|^{2} \leq \underline{\xi} \cdot \underline{A}(\underline{x}) \underline{\xi}, \quad \underline{\xi} \cdot \underline{\underline{A}}(\underline{x}) \underline{\eta} \leq \beta\|\underline{\xi}\|\|\underline{\eta}\|,
$$

for all $\xi, \eta \in \mathbb{R}^{3}$, and for all $x \in P^{\delta}$. The norm $\|\cdot\|$ is the Euclidian norm in $\mathbb{R}^{3}$. Note that the heterogeneity is in the horizontal direction. This model mimics a plate with transverse inclusions.

We next describe the contents of this paper. In Section 2., we derive our hierarchical model. The resulting equations depend on two small parameters, the thickness and the length scale of the heterogeneity, and pose nontrivial numerical challenges $[2,3,4]$. Next, in Section 3., we state that our model is asymptotically consistent, i.e., it converges in a proper sense to the solution of the original problem as the plate thickness goes to zero. Modeling error estimates come by after a somewhat lengthy asymptotic analysis of both the exact and approximate solution. We present only the results, without proofs. We describe finite elements methods of multiscale type in Section 4., and perform computational experiments using the Residual Free Bubbles (RFB) Method and the Multiscale Finite Element Method in Section 5..

\section{Derivation of the Model}

To derive our model we first note that if $V\left(P^{\delta}\right)=\left\{v \in H^{1}\left(P^{\delta}\right):\left.v\right|_{\partial P_{L}^{\delta}}=0\right\}$, then $u^{\delta}=\underset{v \in V\left(P^{\delta}\right)}{\arg \min } \mathcal{I}(v), \quad$ where $\mathcal{I}(v)=\frac{1}{2} \int_{P^{\delta}} \underline{\nabla} v \cdot \underline{A} \underline{\nabla} v d \underline{x}-\int_{P^{\delta}} f^{\delta} v d \underline{x}+\int_{\partial P_{ \pm}^{\delta}} g^{\delta} v d \underline{x}$.

Our model solution $\tilde{u}^{\delta}$, which approximates $u^{\delta}$, is defined as the minimizer of the potential energy in the space of functions of $V\left(P^{\delta}\right)$ which are linear in the transverse direction, i.e.,

$$
\tilde{u}^{\delta}=\underset{v \in V_{1}\left(P^{\delta}\right)}{\arg \min } \mathcal{I}(v)
$$


and $\left.V_{1}\left(P^{\delta}\right)=\left\{v \in V\left(P^{\delta}\right): v\left(\underset{\sim}{x}, x_{3}\right)=v_{0}(\underset{\sim}{x})+x_{3} v_{1} \underset{\sim}{x}\right), v_{0}, v_{1} \in H_{0}^{1}(\Omega)\right\}$.

If we write

$$
\tilde{u}^{\delta}\left(\underset{\sim}{x}, x_{3}\right)=w_{0}(\underset{\sim}{x})+x_{3} w_{1}(\underset{\sim}{x}),
$$

then $w_{0}, w_{1} \in H_{0}^{1}(\Omega)$ solve

$$
\begin{gathered}
\left.-2 \delta \operatorname{div}[\underset{\sim}{a} \underset{\sim}{x}) \underset{\sim}{\nabla} w_{0}\right]=\int_{-\delta}^{\delta} f^{\delta}\left(\underset{\sim}{x}, x_{3}\right) d x_{3}+g^{\delta}(\underset{\sim}{x}, \delta)+g^{\delta}(\underset{\sim}{x},-\delta) \quad \text { in } \Omega, \\
-\frac{2 \delta^{3}}{3} \operatorname{div}\left[\underset{\sim}{a} \underset{\sim}{(x)} \underset{\sim}{\nabla} w_{1}\right]+2 \delta a_{33}(\underset{\sim}{x}) w_{1} \\
=\int_{-\delta}^{\delta} f^{\delta}\left(\underset{\sim}{x}, x_{3}\right) x_{3} d x_{3}+\delta\left[g^{\delta}(\underset{\sim}{x}, \delta)-g^{\delta}(\underset{\sim}{x},-\delta)\right] \quad \text { in } \Omega, \\
w_{0}=w_{1}=0 \quad \text { on } \partial \Omega .
\end{gathered}
$$

\section{Modeling Error Estimate}

In this section we estimate the modeling error with respect to the thickness $\delta$. This becomes nontrivial since the domain itself depends on $\delta$. Thus we scale the domain to remove such dependence, and compare the solutions in a plate with fixed thickness. Let $P=\Omega \times(-1,1)$. Making the change of coordinates $\underset{x}{\hat{x}}=\left(\underset{\sim}{x}, \delta^{-1} x_{3}\right)$, and defining

$$
u(\delta)(\underline{\hat{x}})=u^{\delta}(\underline{x}), \quad f(\underline{\hat{x}})=f^{\delta}(\underline{x}), \quad g(\underline{\hat{x}})=\delta^{-1} g^{\delta}(\underline{x}),
$$

it follows that

$$
\begin{gathered}
\operatorname{div}[\underset{\sim}{a}(\underset{\sim}{\hat{x}}) \underset{\sim}{\nabla} u(\delta)]+\delta^{-2} \partial_{3}\left[a_{33}(\underset{\sim}{\hat{x}}) \partial_{3} u(\delta)\right]=-f \quad \text { in } P, \\
u(\delta)=0 \quad \text { on } \partial P_{L}, \quad \delta^{-1} a_{33}(\underset{\sim}{\hat{x}}) \partial_{3} u(\delta)=\delta \hat{x}_{3} g \quad \text { in } \partial P_{ \pm} .
\end{gathered}
$$

Assuming that $f$ and $g$ are $\delta$-independent, it is possible to show [14] that

$$
u(\delta) \sim \omega_{0}+\delta^{2} u_{2}-\delta^{2} U_{2}+\delta^{4} u_{4}-\delta^{4} U_{4}+\cdots,
$$

where

$$
\begin{gathered}
\left.\partial_{3}\left[a_{33}(\underset{\sim}{\hat{x}}) \partial_{3} u_{2}\right]=-f-\operatorname{div}[\underset{\sim}{a} \underset{\sim}{\hat{x}}) \underset{\sim}{\nabla} \omega_{0}\right], \\
\left.\partial_{3}\left[a_{33}(\underset{\sim}{\hat{x}}) \partial_{3} u_{2 k}\right]=-\operatorname{div}[\underset{\sim}{a} \underset{\sim}{\hat{x}}) \underset{\sim}{\nabla} u_{2 k-2}\right] \quad \text { for all } k \geq 2 .
\end{gathered}
$$

The boundary conditions on $\partial P_{ \pm}$are

$$
a_{33}(\underset{\sim}{\hat{x}}) \partial_{3} \omega_{0}=0, \quad a_{33}(\underset{\sim}{\hat{x}}) \partial_{3} u_{2}=\hat{x}_{3} g, \quad a_{33}(\underset{\sim}{\hat{x}}) \partial_{3} u_{2 k}=0 \text { for all } k \geq 2 .
$$

and the following compatibility conditions holds

$$
\int_{-1}^{1} u_{2 k}\left(\underset{\sim}{\hat{x}}, \hat{x}_{3}\right) d \hat{x}_{3}=0
$$

Note that the first term in the asymptotic expansion matches $w_{0}$, solution of (2.2). 
The above equations define a sequence of Neumann problems with respect to $\hat{x}_{3}$ in $(-1,1)$, parameterized by $\hat{x} \in \Omega$. From the Dirichlet condition in $(3.1)$, we would like to have $u_{k}=0$ on $\partial P_{L}$, but note that this does not hold in general, making necessary the introduction of correctors

$$
U \sim \delta^{2} U_{2}+\delta^{4} U_{4}+\cdots
$$

where $U_{2 k} \in H^{1}(P)$ solves

$$
\begin{gathered}
-\delta^{2} \operatorname{div}\left[\underset{\sim}{a}(\underset{\sim}{\hat{x}}) \underset{\sim}{\nabla} U_{2 k}\right]-a_{33}(\underset{\sim}{\hat{x}}) \partial_{33} U_{2 k}=0 \quad \text { in } P, \\
\frac{\partial U_{2 k}}{\partial n}=0 \quad \text { on } \partial P_{ \pm}, \quad U_{2 k}=u_{2 k} \quad \text { on } \partial P_{L} .
\end{gathered}
$$

Next we state some results that are necessary to estimate the modeling error. The constants are generally denoted by $c$, even if they are not the same in different occurrences. These are independent of $\delta$ but might depend on $\alpha, \beta, \Omega$ and also on Sobolev norms of $f$ and $g$. We have then the following classical regularity estimates. See [7] for complete proofs.

The result below follows from classical estimates.

Lemma 3.1. Let $\omega_{0}$ and $u_{2 k}$ be defined as above, for $k \in \mathbb{N}$. Then there exists a constant $c$ such that

$$
\left\|\omega_{0}\right\|_{H^{1}(\Omega)}+\left\|u_{2 k}\right\|_{H^{1}(P)} \leq c .
$$

From the following result, we obtain an estimate for problem (3.4) in the $\|\cdot\|_{H^{1}(P)}$ norm.

Lemma 3.2. Let $F \in L^{2}(P)$ and $\Theta \in H^{1}(P)$ weak solution of

$$
\begin{gathered}
-\delta^{2} \operatorname{div}[\underset{\sim}{a}(\underset{\sim}{\hat{x}}) \underset{\sim}{\nabla} \Theta]-a_{33}(\underset{\sim}{\hat{x}}) \partial_{33} \Theta=F \quad \text { in } P, \\
\frac{\partial \Theta}{\partial n}=0 \quad \text { on } \partial P_{ \pm}, \quad \Theta=0 \quad \text { on } \partial P_{L} .
\end{gathered}
$$

Then there exists a constant $c$ such that

$$
\|\Theta\|_{H^{1}(P)} \leq c \delta^{-2}\|F\|_{L^{2}(P)} .
$$

To estimate (3.3), we consider now the problem of finding $\Psi \in H^{1}(P)$ such that

$$
\begin{gathered}
-\delta^{2} \operatorname{div}[\underset{\sim}{a} \underset{\sim}{(\underset{\sim}{x})} \underset{\sim}{\nabla} \Psi]-a_{33}(\underset{\sim}{\hat{x}}) \partial_{33} \Psi=0 \quad \text { in } P, \\
\frac{\partial \Psi}{\partial n}=0 \quad \text { on } \partial P_{ \pm}, \quad \Psi=w \quad \text { on } \partial P_{L},
\end{gathered}
$$

Lemma 3.3. Let $\Psi$ as in (3.5). Assume also that $\int_{-1}^{1} w\left(\underset{\sim}{\hat{x}}, \hat{x}_{3}\right) d \hat{x}_{3}=0$ on $\partial P_{L}$. Then there exists a constant $c$ such that

$$
\|\Psi\|_{L^{2}(P)} \leq c\left\|\partial_{3} \Psi\right\|_{L^{2}(P)}
$$


Lemma 3.4. Let $\Psi$ be the solution of (3.5), where $w \in W^{1, \infty}(P)$. Then there exists a constant $c$ such that

$$
\|\underline{\nabla} \Psi\|_{L^{2}(P)} \leq c \delta^{-1 / 2}\|w\|_{W^{1, \infty}} .
$$

Using Lemmas 3.3 and 3.4, we have that if $U_{2 k}, k \in \mathbb{N}$, solve (3.3), then

$$
\left\|U_{2 k}\right\|_{H^{1}(P)} \leq c \delta^{-1 / 2} \text {. }
$$

We next estimate the residue $r=u^{\delta}-\left(\omega_{0}+\delta^{2} u_{2}\right)$. We first note that

$$
\begin{gathered}
-\delta^{2} \operatorname{div}[\underset{\sim}{a} \underset{\sim}{(\hat{x})} \underset{\sim}{\nabla} r]-a_{33}(\underset{\sim}{\hat{x}}) \partial_{33} r=\delta^{4} a_{33}(\underset{\sim}{\hat{x}}) \partial_{33} u_{4} \quad \text { in } P, \\
\frac{\partial r}{\partial n}=0 \quad \text { on } \partial P_{ \pm}, \quad r=-\delta^{2} u_{2} \quad \text { on } \partial P_{L} .
\end{gathered}
$$

The following result holds.

Theorem 3.1. Let $r$ as above. Then there exists a constant $c$ such that

$$
\|r\|_{H^{1}(P)} \leq c \delta^{3 / 2} .
$$

The following result presents and estimate for the difference between $u^{\delta}$ and the first term of the asymptotic expansion.

Lemma 3.5. Let $u^{\delta}$ be the solution of (1.1), and $\omega_{0}$ be the solution of (2.2). Then

$$
\left\|u^{\delta}-\omega_{0}\right\|_{H^{1}(P)} \leq c \delta^{3 / 2} .
$$

To estimate the modeling error, we need the following result [1].

Lemma 3.6. Let $w_{1} \in H_{0}^{1}(\Omega)$ be the solution of (2.2). Then

$$
\left\|w_{1}\right\|_{H^{1}(\Omega)} \leq c \delta^{1 / 2}
$$

Note that it is also possible to show the above result by modifying the proofs of Theorem 3.2 and Lemma 3.4.

From Lemma 3.6, we have that $\left\|\delta \hat{x}_{3} w_{1}\right\|_{H^{1}(P)} \leq c \delta^{3 / 2}$. Let

$$
\tilde{u}(\delta)(\underline{\hat{x}})=\tilde{u}^{\delta}(\underline{x})=\omega_{0}(\underset{\sim}{\hat{x}})+\delta \hat{x}_{3} w_{1}(\underset{\sim}{\hat{x}}) .
$$

Thus

$\|\tilde{u}(\delta)-u(\delta)\|_{H^{1}(P)} \leq\left\|\tilde{u}(\delta)-\omega_{0}\right\|_{H^{1}(P)}+\left\|u(\delta)-\omega_{0}\right\|_{H^{1}(P)} \leq c \delta^{3 / 2}+c \delta^{3 / 2} \leq c \delta^{3 / 2}$.

We then have the following result.

Theorem 3.2. Let $u(\delta)$ be the solution for (3.1) and $\tilde{u}(\delta)$ the model solution. Then there exists a constant $c$ such that

$$
\|\tilde{u}(\delta)-u(\delta)\|_{H^{1}(P)} \leq c \delta^{3 / 2} .
$$




\section{Multiscale Numerical Schemes}

The plate problem we consider here has rough coefficients, and the reduced model inherits such characteristic. Thus, although the PDEs in (2.2) are much easier to solve than their original three-dimensional counterparts, they still pose a tough computational challenge. In fact, for highly heterogeneous materials, the coefficients $\underset{\sim}{a}(\cdot)$ and $a_{33}(\cdot)$ can be oscillatory, making the traditional finite element and difference methods almost useless. In the second problem in (2.2) a new difficulty arises since the PDE is singularly perturbed with respect to $\delta$.

To overcome such troubles we employ two finite element methods of multiscale type. We first briefly describe the Residual Free Bubbles (RFB) Method [5, 6, 10], which consists in enriching the usual finite element space of polynomials with bubbles, functions that vanish on the border of each element.

Consider the second order elliptic problem

$$
\begin{aligned}
& \mathcal{L} u=f \quad \text { in } \Omega, \\
& u=0 \quad \text { on } \partial \Omega,
\end{aligned}
$$

where the differential operator $\mathcal{L}$ is defined as below:

$$
\left.\mathcal{L} v=-\operatorname{div}[\underset{\sim}{a} \underset{\sim}{x}) \underset{\sim}{\nabla} v], \quad \mathcal{L} v=-\frac{2 \delta^{3}}{3} \operatorname{div}[\underset{\sim}{a} \underset{\sim}{x}) \underset{\sim}{\nabla} v\right]+2 \delta a_{33}(\underset{\sim}{x}),
$$

cf. (2.2). Let $a: H_{0}^{1}(\Omega) \times H_{0}^{1}(\Omega) \rightarrow \mathbb{R}$, be the bilinear form associated with (4.1).

Let $\mathcal{T}_{h}$ be a regular partition of $\Omega$ into finite elements $K$. Associated with such partition, let $V_{1} \subset H_{0}^{1}(\Omega)$ be the space of continuous piecewise linear functions, and the bubble space

$$
V_{B}=\left\{v \in H_{0}^{1}(\Omega):\left.v\right|_{\partial K}=0 \text { for all } K \in \mathcal{T}_{h}\right\} .
$$

The residual free bubble method consists in applying the Galerkin method in $V_{1} \oplus$ $V_{B}$, i.e, we search for $u_{1}+u_{b}$, where $u_{1} \in V_{1}, u_{b} \in V_{B}$, and

$$
a\left(u_{1}+u_{b}, v_{1}+v_{b}\right)=\left(f, v_{h}\right), \quad \text { for all } v_{1}+v_{b} \in V_{P} \oplus V_{B} .
$$

The basic idea now is to apply a static condensation trick and write $u_{b}$ in terms of $u_{1}$. Testing (4.2) with functions in $V_{P}$ only, we gather that $u_{b}=\mathcal{L}_{*}^{-1} f-\mathcal{L}_{*}^{-1} \mathcal{L} u_{1}$, where $\mathcal{L}_{*}^{-1}: L^{2}(\Omega) \rightarrow V_{B}$ is such that if $v=\mathcal{L}_{*}^{-1} g$, for $g \in L^{2}(\Omega)$, then

$$
\begin{aligned}
& \mathcal{L} v=g \quad \text { in } K, \\
& v=0 \quad \text { on } \partial K,
\end{aligned}
$$

for all $K \in \mathcal{T}_{h}$. Thus

$$
a\left(u_{1}-\mathcal{L}_{*}^{-1} \mathcal{L} u_{1}, v_{1}\right)=\left(f, v_{1}\right)-a\left(\mathcal{L}_{*}^{-1} f, v_{1}\right), \quad \text { for all } v_{1} \in V_{1} .
$$

In terms of finite element implementation, if $\left\{\psi_{i}\right\}_{i=1}^{N}$ is a basis of $V_{1}$, where $\psi_{i}$ are the usual hat functions, we define $\lambda_{i}$ such that

$$
\begin{gathered}
\mathcal{L} \lambda_{i}=0 \quad \text { in } K, \\
\lambda_{i}=\psi_{i} \quad \text { on } \partial K .
\end{gathered}
$$


Next, if $u_{1}=\sum_{i=1}^{N} u_{i} \psi_{i}$, then

$$
\sum_{i=1}^{N} a\left(\lambda_{i}, \psi_{j}\right) u_{i}=\left(f, \psi_{j}\right)-a\left(\mathcal{L}_{*}^{-1} f, \psi_{j}\right), \quad j=1, \ldots, N .
$$

The other method that we used to discretize our model is the Multiscale Finite Element Method (MsFEM) $[11,12,13]$. The MsFEM consists simply in using Galerkin method with the subspace generated by functions $\left\{\lambda_{i}\right\}_{i=1}^{N}$.

\section{Numerical Tests}

We assume here that $\underset{\sim}{a}$ is periodic with respect to $\varepsilon$. We use both the RFB and MsFEM for the operator $-\operatorname{div}[\underset{\sim}{a} \underset{\sim}{(x)} \underset{\sim}{\nabla}(\cdot)]$, and both yield indistinguishable results. We follow [15] and assume $\Omega=(0,1) \times(0,1)$, and

$$
\underset{\approx}{a}\left(x_{1}, x_{2}\right)=\frac{9}{2} \sin \left(2 \pi \epsilon^{-1} x_{1}\right) \cos \left(2 \pi \epsilon^{-1} x_{2}\right)+\frac{11}{2}, \quad f(\underset{\sim}{x})=1 .
$$

We start by computing the basis functions. Figure 1 shows $\psi-\lambda$ for a fixed element $K=[0,1 / 16] \times[0,1 / 16]$, and a subgrid of $128 \times 128$ elements. Then, Figure 2 shows $\lambda$, and Figure 3 shows its level curves.

Next, Figure 4 display the profile at $x=y$ of various approximation solutions, and also an "exact" solution, obtained using a over-refined mesh.

Resumo. Desenvolvemos neste trabalho a derivação de modelos bidimensionais para placas heterogêneas. Além disto, estimamos o erro de modelagem, e resolvemos as equações diferenciais resultantes usando métodos de elementos finitos multiescala.

\section{References}

[1] S.M. Alessandrini, D.N. Arnold, R.S. Falk, A.L. Madureira, Derivation and justification of plate models by variotional methods, in "Plates and shells" (Québec, QC, 1996), pp.1-20, CRM Proc. Lecture Notes, vol. 21, Amer. Math. Soc., Providence, RI, 1999.

[2] D.N. Arnold, A.L. Madureira, Asymptotic estimates of hierarchical modeling, Math. Models Methods Appl. Sci., 13 (2003), 1325-1350.

[3] D.N. Arnold, A.L. Madureira, S. Zhang, On the range of applicability of the Reissner-Mindlin and Kirchhoff-Love plate bending models, J. Elasticity, 67 (2002), 171-185.

[4] F. Auricchio, C. Lovadina, A.L. Madureira, An asymptotically optimal model for isotropic heterogeneous linearly elastic plates, Math. Model. Numer. Anal., 38 (2004), 877-897. 


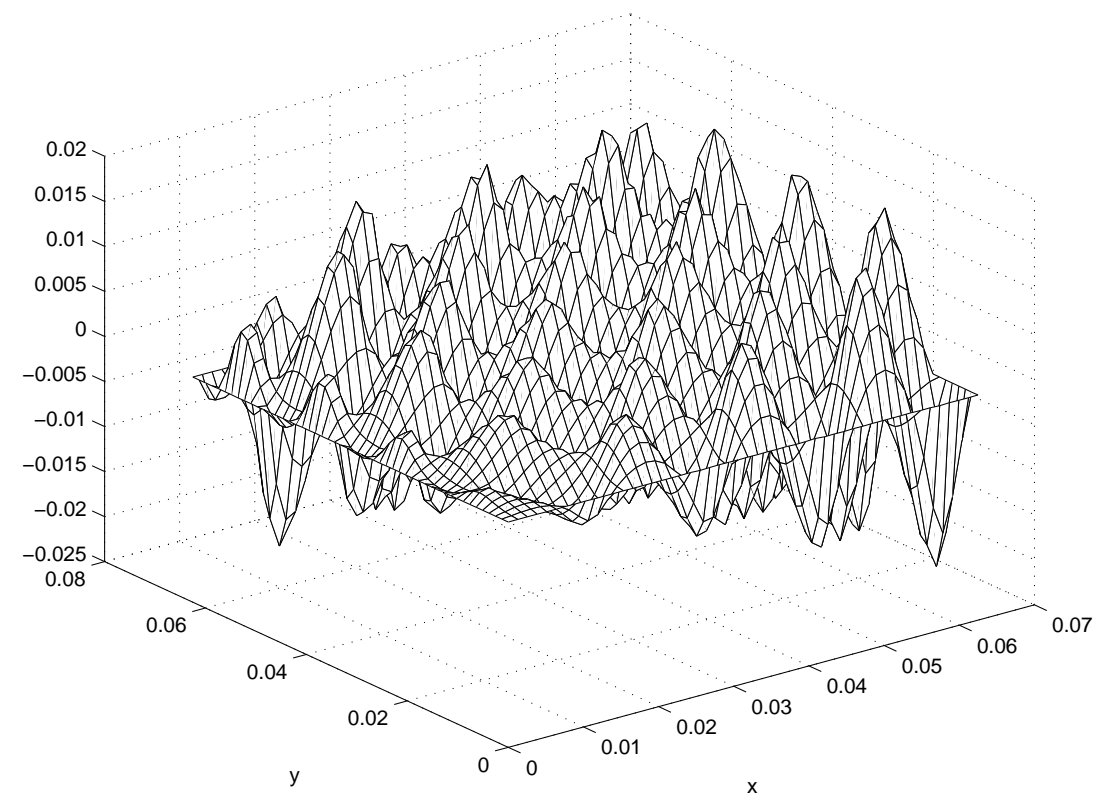

Figure 1: Plot for $\psi-\lambda$.

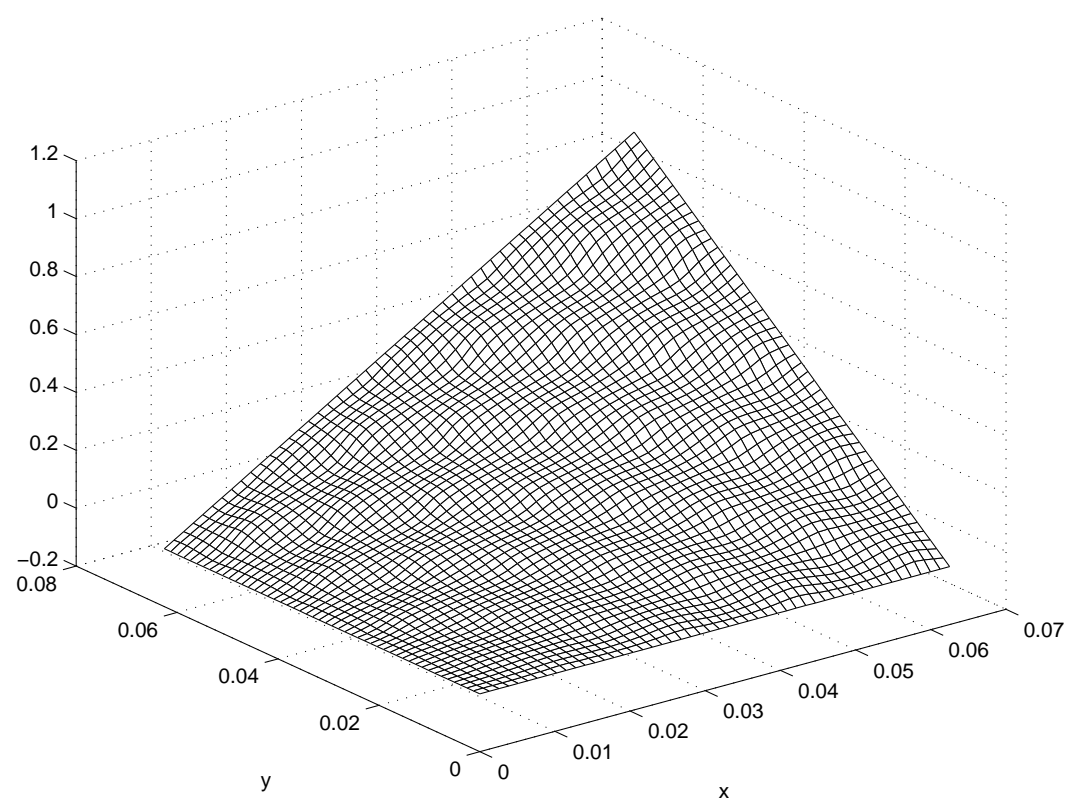

Figure 2: Basis function $\lambda$. 


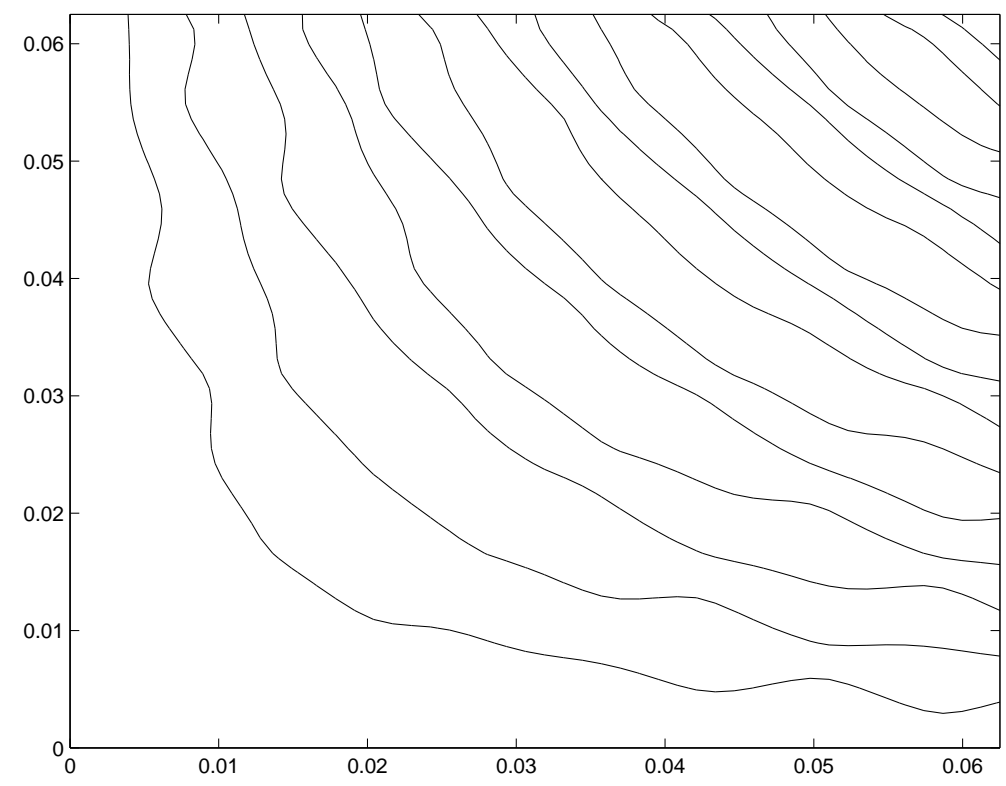

Figure 3: Level curves for $\lambda$.

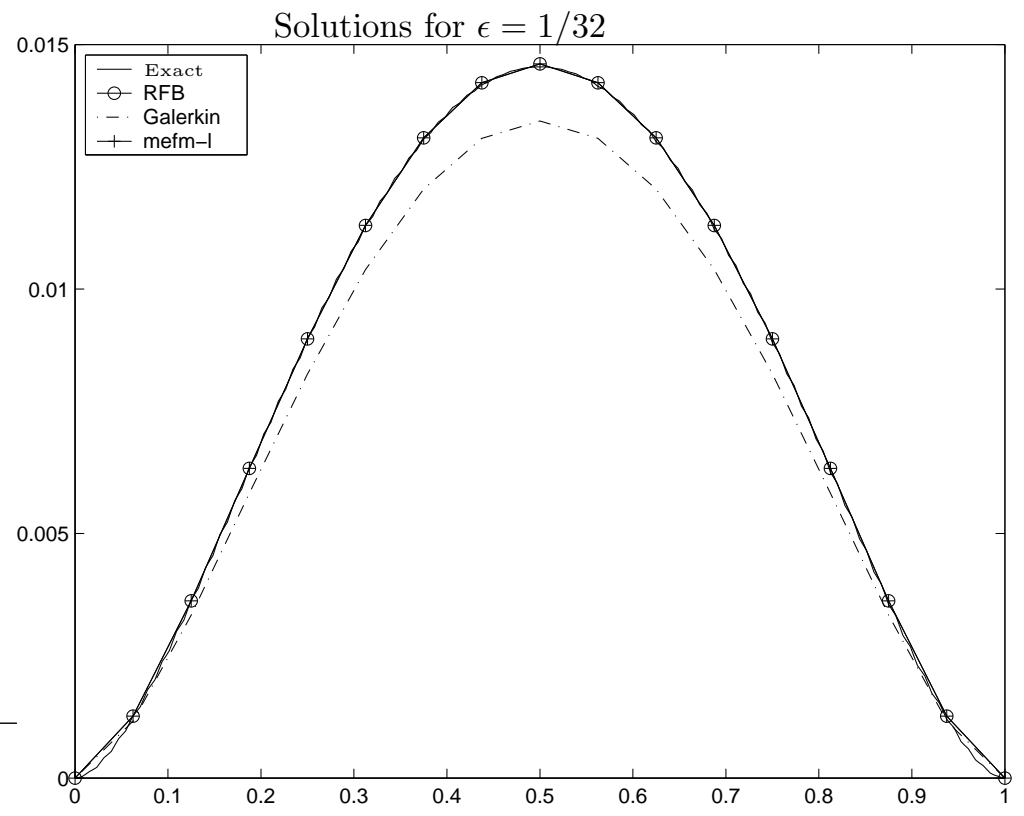

Figure 4: Diagonal profiles for exact, RFB, MsFEM and Galerkin solutions. 
[5] F. Brezzi, A. Russo, Choosing bubbles for advection-diffusion problems, Math. Models Methods Appl. Sci., 4 (1994), 571-587.

[6] F. Brezzi, L.P. Franca, T.J.R. Hughes, A. Russo, $b=\int g$, Comput. Methods Appl. Mech. Engrg., 145 (1997), 329-339.

[7] A.C. Carius, "Modelagem Hierárquica para a Equação do Calor em uma Placa Heterogênea", Dissertação de Mestrado, LNCC, Petrópolis, RJ, 2006.

[8] P.G. Ciarlet, "Mathematical Elasticity, vol I", Studies in Mathematics and its Applications, North-Holland Publishing Co., Amsterdam, 1998.

[9] D. Cioranescu, J.S.J. Paulin, "Homogenization of Reticulated Structures", Applied Mathematical Sciences, Springer-Verlag, New York, 1999.

[10] L.P. Franca, An overview of the residual-free bubbles method, in "Numerical Methods in Mechanics" (Concepción, 1995), pp.83-92, Pitman Res. Notes Math., Longman, Harlow, 1997.

[11] T.Y. Hou, Numerical approximations to multiscale solutions in partial differential equations, in "Frontiers in Numerical Analysis" (Durham, 2002), pp. 241-301, Universitext, Springer, Berlin, 2003.

[12] T.Y. Hou, X. Wu, Z. Cai, Convergence of a multiscale finite element method for elliptic problems with rapidly oscillating coefficients, Math. Comp., 68 (1999), 913-943.

[13] T.Y. Hou, X. Wu, A multiscale finite element method for elliptic problems in composite materials and porous media, J. Comput. Phys., 134 (1997), 169-189.

[14] A.L. Madureira, Hierarchical modeling based on mixed principles: asymptotic error estimates, Math. Models Methods Appl. Sci., 15 (2005), 985-1008.

[15] G. Sangalli, Capturing small scales in elliptic problems using a residual-free bubbles finite element method, Multiscale Model. Simul., 1 (2003), 485-503. 